\title{
The operation of a 3D wave basin in force control
}

\author{
Johannes Spinneken and Chris Swan \\ Imperial College London, Department of Civil and Environmental Engineering \\ London SW7 2AZ
}

2012

\begin{abstract}
The present study concerns the generation and absorption of directional waves in a facility where the generation and absorption mechanism is based on force-feedback control. Many laboratories worldwide are now equipped with such technology, and the world's largest wave basin (the David Taylor Basin at the Naval Surface Warfare Centre, Carderock, USA) is currently being refurbished utilising force-driven wavemakers.

Traditionally, the wave generation in such facilities was based on an empirical transfer function. In contrast, an entirely analytic approach is presented herein. A theoretical transfer function, enabling fully deterministic wave generation in force-controlled waves basins, is derived for the first time. The theory is applicable to both flap- and piston-type wave machines. Even though the present study focuses on flap-type geometries, the results are readily adopted to the piston case.

To demonstrate the successful application of the novel transfer function, a substantial experimental study is presented. As part of the experimental work, a direct comparison to an empirical transfer function is made and the benefits of either approach are discussed. Overall, very good agreement between the expected wave field and the experimental data is shown. However, some departures remain, particularly for highly directional waves and broad-banded spectra.
\end{abstract}

Keywords: Laboratory wave generation, Wavemaker theory, Force-feedback control, Multi-directional waves, Wave basin

\section{Introduction}

In the context of wave making, the ultimate goal of any laboratory model testing apparatus must be the simulation of the ocean at scale. Even though many studies are performed in 2-dimensional (2D) wave flumes, most advanced testing is inevitably undertaken in 3-dimensional (3D) wave basins. Wave flumes are often built as very long channels at reasonable cost; reflections from the beach taking a relatively long time to influence the testing area. In contrast, in multi-directional wave basins it is often the case that after only a few wave periods the testing area is contaminated by waves reflected from the beach. Absorbing these waves on the wavemaker boundary is crucial in order to prevent the built-up of energy in the basin. This is particularly important for long wave components; a regime where beach absorption is inherently poor.

A multi-directional absorbing wave basin was first described by Salter (1982). More recently, the IAHR seminars 'Wave Analysis and Generation in Laboratory Wave Basins' (1987) and 'Multi-directional Waves and their Interaction with Structures' (1997) contributed a large number of publications in the field of multi-directional wave generation, some of which concern absorbing facilities. Notable publications include Mansard et al. (1997), providing a review of multi-directional wave making facilities; Schäffer \& Klopman (1997), reviewing multi-directional absorption strategies (see also Schäffer \& Klopman (2000)); and Hald \& Frigaard (1997), presenting a novel method for the active absorption of multi-directional waves.

For facilities where the control is based upon the position or displacement of the wave board, the theoretical operation has long been established (Biésel \& Suquet 1954, Ursell et al. 1960). In contrast, the present study develops the theoretical aspects of multi-directional wave generation and absorption in a facility where the absorption technique is based on force control, as originally discussed by Salter (1982). The inherent 
differences between a position-control strategy and a force-control mechanism have previously been outlined in ?; the latter contributions being limited to $2 \mathrm{D}$ effects.

In essence the differences between position control and force control lie in the hydrodynamic feedback path that arises in force control. In the position-control case, the generated wave field (both the progressive wave component and the evanescent modes in the vicinity of the wave board) do not feed-back into the generation process. In fact, the evanescent modes are irrelevant for the generation process. These modes only contribute to the wave field adjacent to the wave board and may affect the maximum power rating of the wave board drive system, but do not affect the relation between the control quantity (board displacement) and the process or output quantity (the amplitude of the progressive wave). As a result, the transfer function in position control is often expressed as a wave-amplitude ratio, directly linking the wave board displacement and the amplitude of the generated progressive wave.

In contrast, both the progressive wave component and the evanescent modes affect the generation process in force-feedback control. In this latter case a transfer function is commonly defined as the relationship between the wave-board force and the amplitude of the progressive wave component. Such a transfer function must address the full hydrodynamics of the problem (as established by Biésel \& Suquet (1954)). Moreover, a transfer function must also incorporate the absorption control mechanism (Spinneken \& Swan 2009a, Spinneken \& Swan 2011). At present, a theoretical transfer function enabling deterministic wave generation in a 3D force-controlled wave basin does not exist, and will be derived herein for the first time.

Building upon the authors' earlier work, the present study derives the hydrodynamic coefficients for the generation of oblique waves; their derivation being based on the assumption of an infinitely long wavemaker. ${ }^{1}$ In working towards this result, which is presented in $\S 2.3$, it becomes clear that the infinitely long wavemaker assumption places some restrictions on the accuracy of the results. In light of this, $\S 2.1$ argues the need for adopting such a solution and indicates the range of applicability. Moreover, the effects related to a 'snake-type' geometry with a finite wavemaker segment width are investigated in $\S 2.2$.

Based on an optimal 2D absorption strategy for an infinitely long wavemaker, the 3D absorption characteristic is evaluated and the relative merits of applying an advanced 3D strategy are discussed. With the absorption technique having been established, a theoretical transfer function for one particular wave basin is derived. However, the approach adopted is general and could equally be applied to other $3 \mathrm{D}$ wave basins. In particular, it is intended to apply this transfer function to commission the new wavemakers in the David Taylor Basin at the Naval Surface Warfare Centre, Carderock Division (NSWCCD), USA (Hayden et al. 2010). As part of the wavemaker modernisation program at NSWCCD a new wave synthesis tool has been developed (Rogers et al. 2010), and this tool also relies on the correct calibration or operation of the facility.

All the studies noted above are limited to first-order effects. In contrast, Schäffer \& Steenberg (2003) presented a second-order wavemaker theory for multi-directional waves; the combination of this theory with active absorption being stated as a long term goal. However, the authors are unaware of any experimental evidence supporting the use of this multi-directional second-order theory (even excluding active absorption); the absence of data perhaps indicative of the challenges associated with such a generation technique. Similar arguments apply to active absorption in multi-directional wave facilities; very limited experimental evidence of their 3D absorption characteristic having been provided over the past decades. To date, there is only one study that discussed the absorption performance of waves in a 3D environment, but this relates to numerical computations (Newman 2010).

The validity of the theoretical model derived herein is investigated in an accompanying experimental study (§3). This experimental study focuses on the accurate generation of multi-directional waves with active forcefeedback enabled. The ability to simultaneously generate and absorb waves has previously been demonstrated in Spinneken \& Swan (2009b), where a double-ended wave flume was considered. A confirmation of the 3D absorption coefficient is outside the scope of the present study, but is envisaged for future work.

All experiments shown herein were conducted in a multi-directional wave basin located in the Hydrodynamics Laboratory at Imperial College London (ICL). The wavemaker at ICL consists of 56 individual

\footnotetext{
${ }^{1}$ In the context of the present study the term 'wavemaker' refers to the entire wave generating boundary and not to individual segments thereof. Moreover, the term 'length' will be used to illustrate the full extent of this boundary. In contrast, the term 'width' refers to the extent of the individual wavemaker segments.
} 
segments and spans a total length of $20 \mathrm{~m}$; the paddle width being approximately $b=0.36 \mathrm{~m}$. The wave basin is operated at a nominal water depth of $h=1.5 \mathrm{~m}$. The wave paddles are of the hinged type, and the hinge is located $0.7 \mathrm{~m}$ below the still water level (equivalent to $0.8 \mathrm{~m}$ above the bed).

The present results show that by incorporating the effects of finite-segment width within the theoretical transfer function, a significant improvement in the experimental data can be achieved (§3.1). At present, the two theories are combined in a somewhat ad-hoc manner; a consistent formulation being envisaged for future work. While the comparison with the ad-hoc approach primarily concerns the amplitude content of random wave spectra, an extensive experimental validation of the phase information is also provided. To achieve this latter task, a number of focused wave events are generated, including both uni-directional and multi-directional (or directionally spread) wave groups (§3.2). Within this aspect of the work, the theoretical transfer function is directly compared to a previously published empirical calibration approach developed in the same facility (Masterton \& Swan 2008). Lastly, the energy content within the wave basin is further observed, and the theoretical transfer function is compared to recent empirical transfer function data for the wave basin at ICL $(\S 3.3)$.

\section{Theoretical Analysis of Directional Wave Generation}

\subsection{Directional Wavemaker of Finite Length}

A theory describing finite-length multi-directional wavemakers was developed by Dalrymple (1985) and summarised in Naito (2006). To identify the effective basin usage area, appropriate to the experimental study presented in $\S 3$, a brief review is included here. The wavemaker is oriented along the $y$-axis and the waves propagate in the positive $x$-direction; the $x$-axis being bound by $x=0$, where the wavemaker is located, and $x=+\infty$. The vertical $z$ coordinate is measured upwards from the still water level, with $z=-h$ describing a uniform bed of constant depth.

For simplicity, side wall reflection is neglected and the basin extents to $y= \pm \infty$. Within this infinite basin, a finite wavemaker of length $2 \beta$ is located between $-\beta \leq y \leq \beta$. Furthermore, the contours of the wave crests and the $y$-axis define an angle $\alpha$, and the wave direction vector $\vec{k}$ may be expressed by $\vec{k}=\left(k_{x}, k_{y}\right)=k(\cos \alpha, \sin \alpha)$. The wave length of the progressive wave is denoted by $L_{0}$. The schematic given in Figure 1 illustrates the layout of a 3D wave basin, defining the terms involved. At this stage it is important to note that the wavemaker is modelled as a continuous function; aspects relating to the finite segment width being addressed in $\S 2.2$.

Following Dalrymple (1985), the progressive part of the velocity potential in this domain may be expressed as

$$
\boldsymbol{\Phi}_{\boldsymbol{p}}(x, y, z, t)=\Re\left\{-\frac{i R}{2} \cosh k(h+z) e^{-i \omega t} \int_{-\beta}^{\beta} e^{i k_{0 y} \zeta} H_{0}^{(1)}\left[k \sqrt{x^{2}+(y-\zeta)^{2}}\right] d \zeta\right\},
$$

where $H_{0}^{(1)}$ is the Hankel function of the first kind and $R$ is a geometry dependent coefficient. The exact value of $R$ is irrelevant, as the representation of the wave field presented below will be normalised. Furthermore, the angular wave frequency, $\omega$, and the wavenumber, $k$, are related by the well known dispersion equation

$$
\omega^{2}=g k \tanh k h,
$$

where $g$ is the acceleration due to gravity. Based on the expression of the velocity potential in equation (1), the surface elevation in the wave basin may be computed.

Figure 2 shows contour plots of the surface elevation within a section of this infinite wave basin. Only the progressive wave component is shown; the evanescent modes having been neglected. In obtaining the data shown in Figure 2, the water depth was set to $h=1.5 \mathrm{~m}$ and a wavemaker of length $2 \beta=20 \mathrm{~m}$ is located between $-10 \mathrm{~m} \leq y \leq+10 \mathrm{~m}$. This specific geometry was chosen as this corresponds to the dimensions of the ICL wave basin; experimental data relating to this facility being presented in $\S 3$. Once again, at this stage the finite wavemaker consists of indefinitely small segments. The normalised surface elevations shown in Figure 2 are based on two different propagation directions: (a) $\alpha=15^{\circ}$ and (b) $\alpha=45^{\circ}$. The wave frequency is held constant at $f=1.0 \mathrm{~Hz}$.

Observing Figure 2, the normalised surface elevation defines a regular wave train propagating in a given direction. Dark areas indicate the instantaneous location of a wave crest, whereas light areas indicate the 
location of a wave trough. Due to the effect of wave diffraction, the wave field is inhomogeneous. As such, only part of the area of the wave basin may be used for model testing. This is particularly evident for the wave case shown in sub-plot (b), corresponding to $\alpha=45^{\circ}$. In this case, approximately two thirds of the basin is available for testing. Careful observation of Figure 2 (b) shows that a notable variation of elevation occurs along the crests which needs to be taken into account. In this regard, it is also important to note that the theoretical model does not include any side wall reflections which would further reduce the testing area. Nevertheless, the role of diffraction and the effective basin usage area are now clearly identified.

\subsection{Segmented-type Wave Generators}

In reality, a wavemaker will consist of individual segments of finite width and the sinusoidal velocity distribution along the $y$-axis, appropriate to the generation of oblique waves, is approximated by a piecewise constant function. This wavemaker motion is illustrated in Figure 1. Using the notation defined in Figure 1, it is clear that

$$
\sin \alpha=\frac{\lambda_{0}}{N b},
$$

where $b$ is the individual wave board width and the integer $N$ is the number of wave boards in one oscillation in the $y$-direction. The demand signal phase shift between two adjacent wavemakers is thus given by

$$
\varphi=\frac{2 \pi}{N}
$$

and equation (3) may be rewritten as

$$
\sin \alpha=\frac{\varphi \lambda_{0}}{2 \pi b}
$$

In seeking an explanation for the existence of spurious waves when using finite width paddles, Sand \& Mynett (1987) derived an expression for the wave modes created by a segmented-type wavemaker. Their result has a similar form to equation (5) and is given by

$$
\sin \alpha_{p}=\frac{(\varphi-2 \pi p) \lambda_{0}}{2 \pi b},
$$

where $p$ is an integer with the discrete values of $0, \pm 1, \pm 2 \ldots$; a full derivation of this result being given by Sand (1979). The mode $p=0$ corresponds to the desired wave component (equation (6) reducing to equation (5)) and values of $|p|>0$ represent the unwanted or spurious waves modes. Sand \& Mynett (1987) also showed that all valid values of $p$ must lie within the interval

$$
\frac{\varphi}{2 \pi}-\frac{b}{\lambda_{0}}<p<\frac{\varphi}{2 \pi}+\frac{b}{\lambda_{0}}
$$

Furthermore, the ratio of the Fourier amplitudes of the wavemaker position signal was derived by Sand (1979) as

$$
\xi=-\frac{\sin (\pi(p N-1) / N)}{(p N-1) \sin (-\pi / N)},
$$

which is the ratio of mode $p$ divided by mode zero. This ratio applies to constant-element wavemakers, where the velocity between two wavemaker segments changes abruptly (Figure 1). Sand (1979) also showed that for a linear transition of the velocity between two segments, the ratio of the Fourier amplitudes is given by $\xi^{2}$. In this context it is important to note that Sand (1979) interpreted this ratio as the ratio of wave amplitudes rather than wavemaker position signal. Schäffer (1998) clarified this misunderstanding; the difference between the two becoming critical when a spurious mode is generated in a very oblique direction. Schäffer (1998) also extended the concept of constant and linear elements, and derived an expression for spline-elements.

To demonstrate the effect of a finite paddle width it is instructive to consider the simpler form given by equation (8). Assuming that the wave board width, the wave frequency, the wave propagation angle and the water depth are given by $b=0.36 \mathrm{~m}, f=1.8 \mathrm{~Hz}, \alpha=45^{\circ}$ and $h=1.5 \mathrm{~m}$ respectively, $p$ must lie in the range $-0.22<p<1.27$. Consequently, the only valid values of $p$ are 0 and 1 . Substituting this result into equation (6) gives the two angles of wave propagation. The first corresponds to the desired progressive wave 
at $\alpha_{0}=45^{\circ}$, while the second represents a spurious progressive wave at $\alpha_{1}=-39.2^{\circ}$. Using equation (8), the amplitude of this latter component is found to be $112 \%$ of the primary wave amplitude; an unwanted or spurious wave mode having an amplitude larger than the desired wave is thus generated.

This set of equations is best illustrated as shown in Figure 3; the dimensionless ratio $\lambda_{0} / b$ being plotted against the angle of wave propagation $\alpha$. Note that in contrast to the representation given in Figure 3, Sand \& Mynett (1987) presented a related figure in which the ordinate was scaled by $\sin \alpha$. Observing the occurrence of spurious wave modes, four distinct regions may be identified within Figure 3.

i. For values of $\lambda_{0} / b>2$, waves with a propagation direction of up to $\alpha=90^{\circ}$ may be generated and spurious modes do not exist.

ii. For values of $\lambda_{0} / b<2$, the solid line represents the limit of $p=0$. On the right hand side of this line, only the desired progressive mode $(p=0)$ exists.

iii. In between the dashed line and the solid line, one or more spurious modes are generated $(p \geq 1)$. In considering this region, Sand \& Mynett (1987) give a more detailed representation including the number of modes generated.

iv. The dashed line represents the limit of $\varphi=\pi$. In this case, two adjacent wavemakers are exactly out of phase and the spurious wave is of equal magnitude when compared to the desired wave. For all cases on the left hand side of the dashed line, the spurious modes arising due to the finite segment width are larger than the desired wave.

The representation given in Figure 3 is plotted with the dimensionless ratio $\lambda_{0} / b$. In contrast, Figure 4 illustrates the spurious modes associated with the wave basin located in the Hydrodynamics Laboratory at ICL; the calibration of this particular facility being presented by Masterton \& Swan (2008). Given that Figure 4 is plotted in terms of wave frequency $f$, the directional sense of the abscissa is inverted when compared to Figure 3.

Observing Figure 4 in detail, it is important to note that the wave field is free of oblique spurious modes for frequencies $f<1.5 \mathrm{~Hz}$. This is in marked contrast to frequencies in the region of $f \approx 2 \mathrm{~Hz}$. In this latter case, the spurious wave modes may be as large as the desired primary wave for propagation angles as small as $\alpha=35^{\circ}$.

Figure 5 shows the calibration data for the wave basin at ICL based on the empirical results presented in Masterton \& Swan (2008). The calibration curves for $\alpha=0^{\circ}$ and $\alpha= \pm 40^{\circ}$ are shown, and these are expressed in terms of the tank transfer function $T_{f}$. For a discussion concerning the unit system of $T_{f}$ the reader is referred to Spinneken \& Swan (2011). In addition to these calibration curves, the limits for $p=0$ (solid line in Figure 4) and $\varphi=\pi$ (dashed line in Figure 4) are provided for a wave propagation angle of $\alpha= \pm 40^{\circ}$. Given the above noted discussion, the calibration results for $\alpha= \pm 40^{\circ}$ and frequencies on the right hand side of these lines must be questioned, particularly those on the right hand side of the dashed line representing $\varphi=\pi$.

\subsection{Infinitely Long Wavemaker}

The following discussion presents the linear theory for an infinitely long wavemaker with no active absorption; the velocity distribution along the wavemaker being assumed to vary as a continuous function. The derivation of the hydrodynamic coefficients and the theoretical force transfer function will be based upon this theory. However, the effects introduced by a wavemaker of finite length and finite segment width will be discussed further within the experimental validation of the force transfer function given in $\S 3$.

For brevity, the complete formulation is omitted here; the reader being referred to Schäffer \& Steenberg (2003) for further details. Adopting a formulation and notation similar to that presented by Schäffer \& Steenberg (2003), the first-order velocity potential is given by

$$
\Phi^{(1)}(x, y, z, t)=\frac{1}{2}\left\{\frac{i g X_{a}}{\omega} \sum_{j=0}^{\infty} e_{j} \frac{\cosh k_{j}(z+h)}{\cosh k_{j} h} e^{i\left(\omega t-\vec{k}_{j} \vec{x}\right)}+\text { c.c. }\right\} .
$$


This result relies on the paddle motion at still water level being defined as

$$
X_{0}^{(1)}=\frac{1}{2}\left\{-i X_{a} e^{i\left(\omega t-k_{0 y} y\right)}+c . c .\right\}
$$

where $X_{a}$ is the complex amplitude of the wave board motion and c.c. denotes the complex conjugate of the preceding term. Within the solution for the velocity potential the index $j=0$ refers to the progressive wave mode, whereas that part of the solution corresponding to $j \geq 1$ describes an infinite series of evanescent wave modes. These evanescent modes arise in the vicinity of the wave board and are due to the mismatch between the wave board velocity and the fluid velocity under a progressive wave train.

The wave number vector $\vec{k}_{j}=\left(k_{j x}, k_{j y}\right)=k_{j}\left(\cos \alpha_{j}, \sin \alpha_{j}\right)$ for the progressive wave mode with $j=0$ is identical to that defined in Figure 1. Comparing this latter solution for the velocity potential (equation (9)) to that for the wavemaker of finite length (equation (1)), the benefits in terms of ease of solution are evident.

Following Schäffer \& Steenberg (2003), the solution for the wave field coefficients appropriate to the generation of oblique waves, $e_{j}$, is readily shown to be

$$
e_{j}=\frac{1}{\cos \alpha_{j}} c_{j},
$$

where the coefficients $c_{j}$ are the solution to the $2 \mathrm{D}$ problem given by

$$
c_{j}=\sinh \left(k_{j} h\right) \frac{\Lambda_{1}\left(k_{j}\right)}{\Lambda_{2}\left(k_{j}\right)},
$$

where

$$
\Lambda_{1}\left(k_{j}\right) \quad=\quad \sinh \left(k_{j} h\right) \quad-\frac{d+l}{h+l} \sinh \left(k_{j} d\right) \quad+\quad \frac{1}{h+l} \frac{\cosh \left(k_{j} d\right)-\cosh \left(k_{j} h\right)}{k_{j}}
$$

and

$$
\Lambda_{2}\left(k_{j}\right)=\frac{1}{2}\left[k_{j} h+\sinh \left(k_{j} h\right) \cosh \left(k_{j} h\right)\right] .
$$

The quantities $d$ and $l$ define the wavemaker geometry; two quantities being required to define the theory for both flap- and piston-type wavemakers in a single compact notation. The length $l$ gives the centre of rotation, and $l=\infty$ for a piston-type wavemaker. The reader is referred to Schäffer \& Steenberg (2003) for further examples concerning the correct application of $d$ and $l$. Furthermore, $\alpha_{j}$ is the direction of wave propagation generalised to complex wave modes. The expression for $\cos \alpha_{j}$ for the general complex case may be rewritten as

$$
\cos \alpha_{j}=\sqrt{1-\frac{k^{2} \sin ^{2} \alpha}{k_{j}^{2}}}= \begin{cases}\cos \alpha \leq 1 & \text { for } j=0 \\ \sqrt{1+\frac{k^{2} \sin ^{2} \alpha}{\left|k_{j}\right|^{2}}} \geq 1 & \text { for } j>0 .\end{cases}
$$

Observing this latter representation for $\cos \alpha_{j}$, it is important to note that for the real solution $(j=0)$ the expression simply evaluates to $\cos \alpha_{0}$ which is always less than or equal to one. As a result, the coefficient for the generation of oblique waves, $e_{0}$, is larger than $c_{0}$. The relation between the amplitude of the paddle motion, $X_{a}$, and the amplitude of the oblique progressive wave mode is

$$
A=e_{0} X_{a}
$$

from which it is clear that, for a particular wave board amplitude, the progressive wave amplitude increases in the oblique case; the factor of proportionality being $1 / \cos \alpha$ for all frequencies. A physical explanation for this dependency is given in $\S 2.4$ where the hydrodynamics coefficients (added mass and radiation damping) are discussed. At this stage it is important to note that in the remainder of this study the wave propagation angle $\alpha$ refers to the propagation direction of the progressive wave mode, the subscript 0 (as in $\alpha_{0}$ ) being omitted.

The generation of the evanescent wave modes $(j>0)$ needs further consideration. For this purpose, the first-order coefficients for a range of wave propagation directions are presented in Figure 6 (a) and (b), concerning piston-type and flap-type wave machines respectively; the latter defined by $d / h=0.5$. In both figures the coefficient for the progressive wave mode, $e_{0}$, and the infinite sum $i \sum_{j=1}^{\infty} e_{j}$ are shown. 
As noted above, the progressive wave amplitude for oblique waves obeys a $1 / \cos \alpha$ relationship when compared to the 2D solution. This is particularly evident within Figure 6 (a) where the deep water limit of $c_{0}=2\left(e_{0}=c_{0}\right.$ for $\left.\alpha=0^{\circ}\right)$ increases to $e_{0}=4$ for $\alpha=60^{\circ}$. On the contrary, considering the sum of the evanescent modes $(j=1 . . \infty)$, it is clear that their amplitude decreases with increasing wave propagation angle (Figure 6 (a) and (b)). This decrease is consistent with the findings presented in Schäffer \& Steenberg (2003) and Newman (2010). For example, the magnitude of the evanescent mode sum for the piston-type machine shown in Figure 6 (a) decreases from $i \sum_{j=1}^{\infty} e_{j} \approx 2\left(\alpha=0^{\circ}\right)$ to $i \sum_{j=1}^{\infty} e_{j} \approx 0.7\left(\alpha=60^{\circ}\right)$ for $h / L_{0}=1.8$. As a result, the ratio of $\left(i \sum_{j=1}^{\infty} e_{j}\right) / e_{0}$ reduces significantly when generating oblique waves. This directly affects the hydrodynamic coefficients and hence the machine's absorption characteristic and the first-order force transfer function. It should be noted that the increase in $e_{0}$ is anticipated and easily argued on physical grounds. In contrast, the decrease in the evanescent mode sum is far less intuitive and needs careful consideration.

\subsection{Hydrodynamic Coefficients}

Spinneken \& Swan (2009c) discussed optimum absorption strategies for wavemaking devices and showed that the added mass and the hydrodynamic damping are crucial when adopting a force-based absorption approach. They also derived analytic expressions for these coefficients given a $2 \mathrm{D}$ velocity potential. The only difference arising between the $2 \mathrm{D}$ case and the $3 \mathrm{D}$ case is that the first-order wave field coefficients $\left(c_{j}\right)$ must be replaced by the coefficients appropriate to the generation of oblique waves, $e_{j}$. Apart from this difference, the equations for the hydrodynamic coefficients (equations (16)-(19) in Spinneken \& Swan $(2009 c)$ ) remain unchanged.

If $d(\omega)$ represents the hydrodynamic damping and $m(\omega)$ the added mass the corresponding equations for the piston case are given by

$$
\begin{gathered}
d(\omega)=\frac{\rho g}{\omega} \frac{e_{0}}{k_{0}} \frac{\sinh k_{0} h-\sinh k_{0} d}{\cosh k_{0} h} \\
m(\omega)=\frac{\rho g}{\omega^{2}} \sum_{j=1}^{\infty} \frac{e_{j}}{k_{j}} \frac{\sinh k_{j} h-\sinh k_{j} d}{\cosh k_{j} h}
\end{gathered}
$$

and the flap case by

$$
\begin{aligned}
& d(\omega)=\frac{\rho g(h-d)}{\omega} \frac{e_{0}}{k_{0}^{2}} \frac{k_{0}(h-d) \sinh k_{0} h-\cosh k_{0} h+\cosh k_{0} d}{\cosh k_{0} h} . \\
& =\quad \frac{\rho g(h-d)}{\omega^{2}} \sum_{j=1}^{\infty} \frac{e_{j}}{k_{j}^{2}} \frac{k_{j}(h-d) \sinh k_{j} h-\cosh k_{j} h+\cosh k_{j} d}{\cosh k_{j} h} .
\end{aligned}
$$

Given the discussion relating to the coefficients $e_{j}$, the hydrodynamic damping and the added mass will also vary with the wave propagation angle $\alpha$. Considering first the hydrodynamic damping for the case of oblique wave generation, it is important to note that this term varies with $1 / \cos \alpha$ for all frequencies. This result has also been noted by Newman (2010). The explanation for this lies in the fact that the wave amplitude of the progressive wave component is proportional to $1 / \cos \alpha$. As a result, the radiated energy density is proportional to $1 / \cos ^{2} \alpha$. Observing the component of the group velocity normal to a surface defined by $x=$ constant, this component is proportional to $\cos \alpha$. Consequently, the rate of energy flux or damping must be proportional to $1 / \cos \alpha$. However, in considering such arguments, it is important to note that they only apply to an infinitely long wavemaker.

In contrast, the added mass term does not obey such a simple relationship. Figure 7 illustrates $m(\omega, \alpha)$ for the wave machine geometries discussed earlier; the term $m(\omega, \alpha)$ for a particular wave propagation direction being presented normalised with respect to the added mass in the $2 \mathrm{D}$ case, $m(\omega, 0)$. The curves shown in both figures correspond to a variety of non-dimensional water depths $h / L_{0}$.

In the piston case (Figure $7(\mathrm{a})$ ), values of $h / L_{0}=0.1,0.3$ and 0.5 are considered. The dependency of the added mass term on the wave propagation direction increases significantly in the intermediate water depths $\left(h / L_{0}=0.3\right.$ and 0.5$)$. In the case of $h / L_{0}=0.5$, the value for the added mass is less than $75 \%$ of 
the equivalent $2 \mathrm{D}$ value for $\alpha \geq 40^{\circ}$. In relatively shallow water $\left(h / L_{0}=0.1\right)$, the reduction is substantially smaller.

For the flap-type wave machine $(h / d=0.5)$ considered in Figure $7(\mathrm{~b})$, values of $h / L_{0}=0.1,0.5$ and 1.0 are considered. In this case it is interesting to note that the ratio $m(\omega, \alpha) / m(\omega, 0)$ for $h / L_{0}=1.0$ is larger than the equivalent ratio for $h / L_{0}=0.5$. The origins of this characteristic are best understood by considering the first-order coefficients, $e_{j}$, in Figure 6 (b). In contrast to piston-type wave machines, the infinite sum $i \sum_{j=1}^{\infty} e_{j}$ is negative for small values of $h / L_{0}$ and has a zero crossing at $h / L_{0} \approx 0.6$.

\subsection{Absorption Characteristics}

The variation of the added mass and hydrodynamic damping terms with the angle of wave propagation affects the wave machine's absorption characteristic. The power absorption coefficient, $\gamma$, is defined as the ratio of the absorbed power over the maximum power (Falnes 2002), given by

$$
\gamma=\frac{P_{a}}{P_{a, \max }}=4 \frac{R_{f} R_{d}}{\left(R_{f}+R_{d}\right)^{2}+\left(X_{f}+X_{d}\right)^{2}},
$$

where $R_{d}$ and $X_{d}$ are the real and imaginary part of the dynamic system and $Z_{f}=R_{f}+i X_{f}$ characterise the wave absorption controller. The real part of the dynamic system, $R_{d}$, directly relates to the radiation damping, whereas the imaginary part, $X_{d}$, relates to the added mass, the paddle mass, $M$, and the paddle spring stiffness, $k$. The dynamic system impedance is readily shown to be

$$
Z_{d}=R_{d}+i X_{d}=d(\omega)+i[(M+m(\omega)) \omega-k / \omega] .
$$

The absorption controller $\left(R_{f}\right.$ and $\left.X_{f}\right)$ must be optimised to match $R_{d}$ and $-X_{d}$. In deriving the optimum controller a number of approaches may be adopted. All of these approaches have in common that they seek to define a causal controller for a non-causal control problem. This may be achieved by separating the problem into its causal and non-causal components (Naito \& Nakamura 1985, Chatry et al. 1998). Alternatively, an inherently causal controller may be chosen and optimised to fit the non-causal problem in a least-square sense. An infinite impulse response (IIR) function filter is inherently causal, and provides a robust control tool. The performance of such filter in active force-controlled wave absorption has been demonstrated in Spinneken \& Swan (2009c), where a direct comparison to the work by Naito \& Nakamura (1985) and Chatry et al. (1998) was made. All transfer functions presented in the present paper are based on the optimal absorption technique described in Spinneken \& Swan (2009c).

In contrast to the absorption of uni-directional waves, a matching approach appropriate to the absorption of oblique waves would be difficult to establish. Given the variation of $m(\omega)$ and $d(\omega)$ with the angle of incidence $\alpha$, the ideal absorption controller would need to incorporate the angular dependency, adjusting $\left(R_{f}, X_{f}\right)$ accordingly. Some further aspects of directional absorption control have been discussed in Schäffer (1998), building upon the multi-directional absorption theory developed in Schäffer \& Skourup (1996); the latter demonstrating that such theory is best developed in the wavenumber-frequency domain $\left(k_{y}, \omega\right)$. The authors are aware of the potential benefits of multi-directional absorption technique, and this may be subject of future research. However, the present discussion will be limited to a controller optimised for the 2D case.

In the context of laboratory wave absorption it is more common to define the absorption coefficient in terms of wave amplitude; the amplitude absorption coefficient being defined as $\delta=1-\sqrt{1-\gamma}$. Figure 8 shows the theoretical amplitude absorption characteristic for the wave machine installed in the wave basin at ICL. For this facility the nominal water depth is $h=1.5 \mathrm{~m}$ and the hinge is located at $d=0.8 \mathrm{~m}$ above the bed. The three cases shown within Figure 8 represent the absorption characteristic for $\alpha=0^{\circ}, \alpha=30^{\circ}$ and $\alpha=60^{\circ}$; the controller having been optimised for the 2D case $\left(\alpha=0^{\circ}\right)$. Observing the figure in detail, it is clear that the absorption characteristic for oblique waves $\left(\alpha=30^{\circ}\right.$ and $\left.60^{\circ}\right)$ deteriorates for low to intermediate frequencies, but improves for the highest frequencies. Given the nature of the wave field coefficients, the deterioration in the intermediate frequency range may be related to the $1 / \cos \alpha$ behaviour of the damping term; the controller failing to match the real part (damping) of the wave field.

Whilst this remains the case at high frequencies, the decreasing evanescent mode amplitude for oblique waves in this regime (Figure 6 (b)) leads to an improved absorption characteristic. The explanation for this lies in the fact that at $\alpha=0^{\circ}$ the controller fails to match the imaginary part (evanescent modes) for high 
frequencies. However, when considering oblique waves, the amplitude of the evanescent modes decreases and, consequently, the match is improved as is the absorption characteristic. Overall, the 3D absorption characteristic based on a $2 \mathrm{D}$ optimisation appears acceptable.

\subsection{Theoretical Transfer Function}

With the absorption controller having been established, the transfer function for the force-controlled wave machine may be evaluated. This transfer function relates the force demand signal to the amplitude of the progressive wave mode and is complex (real and imaginary or magnitude and phase) in nature. For an ideal force-feedback control loop, the force control transfer function is defined as

$$
e_{f}=e_{0} \frac{1}{i \omega} \frac{1}{Z_{d}+Z_{f}}
$$

where $e_{0}$ is the wave-amplitude ratio in position control (equation ??eq:3d:ej)). This force transfer function has already been discussed in the context of 2D wave flumes (Spinneken \& Swan 2011). In contrast to the 2D scenario, the transfer function defined previously, $c_{f}(\omega)$, must now be evaluated for each direction of wave propagation; the coefficient becoming $e_{f}(\omega, \alpha)$.

The contour plot in Figure 9 illustrates the magnitude of the theoretical first-order transfer function for the wave basin at ICL. The data is presented as a function of both the wave frequency, $f$, and the direction of wave propagation, $\alpha$. Based on the theory for an infinitely long wavemaker, the transfer function is symmetric and, as a result, the gain or magnitude for positive and negative wave propagation directions is identical. Dark areas effectively indicate a large gain between the demand signal (force or torque) and the wave elevation in the wave basin. To correctly evaluate the surface profile at a given location in the wave basin, the phase information also needs to be taken into consideration, and this will be demonstrated in the following section.

\section{Experimental Investigation}

This section investigates the generation of oblique waves in a 3D wave basin. In particular, the generation of a JONSWAP spectrum is discussed where the data is based on four transfer functions:

i. The theoretical first-order force transfer function, $e_{f}(\omega, \alpha)$.

ii. An $a d$-hoc transfer function representing a combination of $e_{f}(\omega, \alpha)$ and the theory for a segmented-type wave generator outlined in $\S 2.2$. In obtaining the $a d$-hoc transfer function, the effect of the gusset (a flexible membrane between two neighbouring wave paddles) must also be considered. For this purpose a modified form of equation (8) was adopted, assuming a linear variation of the velocity between two neighbouring paddles. As a result, the amplitude of the progressive component in the ad-hoc transfer function was altered by a factor of $\xi^{2}$ when compared to $e_{f}(\omega, \alpha)$, (i) above

iii. The empirical transfer function presented by Masterton \& Swan (2008) (hereafter referred to as MS); the amplitude and phase information in this transfer function being based upon the generation of small amplitude focused wave groups.

iv. A recent empirical transfer function for the 3D wave basin. In this latter case, the amplitude information is based upon the generation of a random (JONSWAP) sea state; the phase information in the transfer function being neglected.

All the experiments were undertaken in the wave basin located in the Hydrodynamics Laboratory at ICL. The nominal water depth in this facility is $h=1.5 \mathrm{~m}$. However, in the context of ongoing work within this basin, the water depth was reduced to $h=1.2 \mathrm{~m}$ at the time of the present study. This change in water depth has been incorporated in (i), (ii) and (iv) above. In contrast, the empirical transfer function described by MS, (iii) above, was obtained for $h=1.5 \mathrm{~m}$, and this must be borne in mind when considering the results relating to (iii). However, with most wave components propagating in relatively deep water, the difference between $h=1.25 \mathrm{~m}$ and $h=1.5 \mathrm{~m}$ is insignificant. Indeed, in an attempt to quantify the magnitude of 
the possible error, the theoretical transfer function, $e_{f}(\omega, \alpha)$, has been evaluated for both water depths; the maximum difference in the transfer function gain for the range of interest being less than $1.6 \%$.

The present experimental study seeks to demonstrate the applicability, or success, of the theoretical force transfer function developed within this study. In so doing, three important cases are considered.

1. The amplitude content for a JONSWAP spectrum, the individual wave components having random phase. A direct comparison between (i) and (ii) above is provided in $\S 3.1$.

2. To demonstrate the successful application of the phase information, a number of focused wave events are generated. This includes both uni-directional and multi-directional, or directionally spread, wave events. A direct comparison between (i) and (iii) above is provided in $\S 3.2$

3. In an attempt to broaden our understanding of an empirical calibration approach, where the calibration routine is based on a random phase spectrum, the energy content in the wave basin is further investigated. A direct comparison between (i) and (iv) above is provided in $§ 3.3$.

\subsection{Amplitude Spectra}

The first set of experiments concerns the amplitude content when generating a spectrum with random phases. A uni-directional JONSWAP spectrum with a spectral peak frequency of $f_{p}=51 / 64 \mathrm{~Hz}$, a peak enhancement factor of $\gamma=3.3$ and a significant wave height of $H_{s} \approx 0.02 \mathrm{~m}$ has been adopted for all wave cases; the spectrum being evaluated in the range $16 / 64 \mathrm{~Hz} \leq f \leq 128 / 64 \mathrm{~Hz}$ with a spacing of $f=1 / 64 \mathrm{~Hz}$ between the individual wave components. The measured data were recorded using standard wave gauges located along the centre of the wave basin to minimise any influence related to the finite length of the wavemaker (Figure $2)$.

Figure 10 (a) illustrates the experimental data for this spectrum at a wave propagation angle of $\alpha=0^{\circ}$. Within this figure, the results for the transfer functions (i) and (ii) above are compared to the theoretical target. The curves are based on a reflection analysis (Lin \& Huang 2004) of the data recorded at eight spatial locations $(3.2 \mathrm{~m} \leq x \leq 4.6 \mathrm{~m} ; y=0)$ with individual gauges spaced $0.2 \mathrm{~m}$ apart. For this purpose, data from the time interval $64 \mathrm{~s} \leq t \leq 128 \mathrm{~s}$ was taken. The initial interval of $t=64 \mathrm{~s}$ is regarded to be sufficiently long to ensure that all the wave components have propagated a sufficient distance to ensure that the beach reflections are fully developed. Moreover, to avoid spectral leakage in the frequency domain, the sample window was chosen to be equivalent to the repeat time of the wave spectrum $\left(T_{r}=64 \mathrm{~s}\right)$.

Observing Figure 10 (a) in detail, both the application of the theoretical force transfer function $e_{f}(\omega, \alpha)$ and the $a d-h o c$ approach result in excellent agreement with the target amplitude content; the small departures apparent at the highest frequencies $(1.5 \mathrm{~Hz}<f<1.9 \mathrm{~Hz})$ probably being due to the nonlinear wave-wave interactions between the largest wave components forming the spectral peak. In fact, for a wave propagation direction of $\alpha=0^{\circ}$, the force transfer function and the ad-hoc transfer function are effectively identical. With the two underlying time series relating to two entirely independent experiments, the data shown in Figure 10 (a) provides evidence for the repeatability of the generation process.

Figure 10, sub-plots (b), (c) and (d) illustrate experimental data relating to the same target JONSWAP spectrum propagating at $\alpha=15^{\circ}, \alpha=30^{\circ}$ and $\alpha=45^{\circ}$ respectively. In considering this data, the wave propagation angle $\alpha$ must not be confused with the directional spreading $\sigma_{\alpha}$; at this stage the wave spectra remain uni-directional. Observing the data based on $e_{f}(\omega, \alpha)$ first, the departures between the experimental measurements and the target spectrum increase with the angle of wave propagation. This is particularly noticeable for frequencies exceeding $f \approx 1 \mathrm{~Hz}$; the agreement in the range $1.5 \mathrm{~Hz}<f<2.0 \mathrm{~Hz}$ being poor at large angles (Figure 10 (c) and (d)). Within this latter region, the data based on the ad-hoc approach is in better agreement with the target spectrum. Indeed, even at the largest angle, $\alpha=45^{\circ}$, the $a d-h o c$ solution is in reasonable agreement. This improvement confirms that some of the departures from the target spectrum are related to the effect of the finite segment width; the remaining departures at large values of $\alpha$ probably being due to wave diffraction effects caused by the finite length of the wavemaker. Equation (1) may be used to estimate the departures due to wave diffraction. For the wavemaker under consideration, and a wave case with $f=1.5 \mathrm{~Hz}$ and $\alpha=45^{\circ}$, diffraction would account for an error of approximately $15 \%$ at $(x, y)=(4 \mathrm{~m}, 0)$. This error is comparable to the departures of the data relating to the ad-hoc transfer function (Figure $10(\mathrm{~d})$.) 
Similar arguments apply to experimental data involving a wave propagation direction of $\alpha=-15^{\circ}$, $\alpha=-30^{\circ}$ and $\alpha=-45^{\circ}$ (not shown herein). Overall, the data based upon the ad-hoc approach shows the most consistent agreement with the target spectrum. This is an unsurprising result as the ad-hoc approach combines aspects of two important theories. It remains a challenge for future work to formulate a theory that covers all aspects of the two theories. In particular, this relates to the amplitude of the evanescent modes for a wavemaker of finite width. Nevertheless, it is important to note that the data relating to the theoretical force transfer function alone, $e_{f}(\omega, \alpha)$, also show good agreement for a wide range of wave conditions.

\subsection{Focused Wave Groups}

The previous discussion concerned the amplitude of the generated wave components within the wave basin. In contrast, the present section seeks to demonstrate the correct application of the phase information. For this purpose, a number of focused wave events are considered; the results being directly compared to both the theoretical linear solutions and experimental data based on the empirical calibration of MS. With the routine adopted by MS being optimised for the generation of focused wave events, this latter transfer function is believed to be the most suitable calibration for the present case. However, when considering the data in the figures that follow, the previously mentioned change in water depth must be borne in mind. Furthermore, as part of ongoing maintenance and improvement work within the wave basin at ICL, some of the drive components and force transducers have been replaced since MS published their results. This may also have had a detrimental effect on the optimum calibration.

The generation of realistic large focused wave groups in the laboratory is best achieved by adopting the NewWave formulation established by Tromans et al. (1991), and based upon the earlier work of Lindgren (1970) and Boccotti (1983). In a NewWave spectrum the amplitude of each component is directly proportional to the energy content of the desired spectral shape (as opposed to the square root of the energy content). Once again, a JONSWAP target spectrum has been adopted and, in accordance with MS, the peak frequency and peak enhancement factor have been set to $f_{p}=53 / 64 \mathrm{~Hz}$ and $\gamma=2.5$ respectively. Furthermore, the amplitude sum $A$ is introduced as the linear sum of all wave components; $A=\sum_{n=1}^{N} a_{n}$, where $N$ is the total number of wave components. As a result, in a linear sea state, the water surface elevation at the focal location and time (where all the wave components superimpose) reaches a maximum value of $\eta_{\max }=A$.

\subsubsection{Uni-directional NewWave Groups}

The first set of cases considers a uni-directional NewWave event based upon a JONSWAP spectrum measured and focused at $x=4.0 \mathrm{~m}, y=0$ and $t=32 \mathrm{~s}$. The amplitude sum has been set to $A=12 \mathrm{~mm}$ so that the particle motion remains linear. Figure 11 (a), (b) and (c) illustrate the water surface elevation, $\eta(t)$, for the wave propagation directions $\alpha=0^{\circ}, \alpha=15^{\circ}$ and $\alpha=30^{\circ}$ respectively. Both the data based on the theoretical force transfer function and the empirical calibration after MS are shown. In all three cases and for both transfer functions the agreement between the experimental data and the theoretical (linear) solution is very good providing evidence that: (i) the gain information in the transfer function yields the correct amplitude content and, (ii) the correct application of the phase information leads to the focusing of the components at the desired time and location. However, some small departures remain and are noted as follows.

- In the vicinity of the main event both the empirical transfer function and the theoretical approach appear to provide a near perfect match to the target for most wave cases.

- In contrast, after the occurrence of the largest event $(t \geq 33 \mathrm{~s})$ the agreement between the data based upon the empirical calibration and the linear target becomes less satisfactory. This effect, particularly the contrast between the match in the vicinity of the event and the mismatch at later times, is not fully understood at present and requires further consideration.

- Based on the theoretical force transfer function, the maximum crest elevations, $\eta_{\max }$, in the oblique cases (Figure 11 (b) and (c)) is slightly reduced when compared to the theoretical value. This decrease is most probably related to the finite segment width of the paddle and the resulting reduction in amplitude content (Figure $10(\mathrm{~b}),(\mathrm{c})$ and (d)). 
- For the largest wave propagation angle $\left(\alpha=30^{\circ}\right.$, Figure $\left.11(\mathrm{c})\right)$ the data based upon the theoretical transfer function suggest that some wave components are out of phase; evidence of this being provided by the slightly increased size of the trough and crest immediately following the largest wave crest, $\eta_{\max }$.

Nevertheless, despite these small departures, the agreement is very good for all cases considered.

\subsubsection{Multi-directional New Wave Groups}

To evaluate the directional capabilities of both transfer functions, a multi-directional, or directionally spread, focused NewWave group has also been generated. Within this example the directional spreading is based upon a normal distribution with a standard deviation of $\sigma_{\alpha}=30^{\circ}$. An identical distribution is applied to all frequencies, with individual wave components being generated at discrete intervals of $\Delta \alpha=5^{\circ}$ in the range $-40^{\circ} \leq \alpha \leq+40^{\circ}$. Figure 12 (a) shows the water surface elevation, $\eta(t)$, recorded by a single wave gauge located at the focal position, $x=4.0 \mathrm{~m}$ and $y=0$. As before, the experimental data relating to the two transfer functions is directly compared to the theoretical (linear) solution. Considering the figure in detail, the agreement between theory and experiment is found to be excellent for both transfer functions, (i) and (iii).

To further evaluate the directional capabilities of the ICL wave basin, a focused wave event identical to that above $\left(f_{p}=53 / 64 \mathrm{~Hz}, \gamma=2.5, A=12 \mathrm{~mm}\right.$ and $\left.\sigma_{\alpha}=30^{\circ}\right)$ was generated at $(x, y)=(4.0 \mathrm{~m},-1.2 \mathrm{~m})$ and $(x, y)=(4.0 \mathrm{~m},+1.2 \mathrm{~m})$; the data being presented in Figure 12, sub-plots (b) and (c) respectively. As before, the agreement between the experimental data and the linear theoretical solution is very good.

The directional spreading of the event illustrated in Figure 12 (a) is further illustrated by considering the transverse variation in the crest elevation, $\eta_{\max }$, at the focal time $(t=32 \mathrm{~s})$, and this is indicated in Figure 13. For this purpose, nine wave gauges were located at $x=4.0 \mathrm{~m}$ and $-1.2 \mathrm{~m} \leq y \leq 1.2 \mathrm{~m}$ with a spacing of $\Delta y=0.3 \mathrm{~m}$ between individual gauges. Overall, the agreement between the measured data and the linear prediction is good for both transfer functions. Indeed, the only significant departures between the two approaches is suspected to relate to a slight positioning error $(\Delta x=0.1 \mathrm{~m})$ in the calibration routine adopted by MS.

The ultimate goal of any experimental investigation concerning focused groups must be the correct generation of a very large wave event. The NewWave event shown in Figure 14 is based on a linear amplitude sum of $A=58 \mathrm{~mm}$; all other parameters being identical to the spectrum considered above. As before, the experimental data are compared to the linear theoretical solution. In this context it is important to note that, due to nonlinear wave-wave interactions, the linear solution fails to accurately model the water surface elevation. Nevertheless, the general agreement between the experimental data and the linearly predicted surface profile suggests that the two transfer functions are indeed appropriate to the generation of large focused events; a detailed analysis of the higher order wave content being outside the scope of the present study. Most importantly, the agreement between the experimental data based on the theoretical transfer function and the data generated using the empirical transfer function outlined in MS, suggests that the former is at least as good as the latter and avoids the need for extensive empirical calibrations.

\subsection{Energy Spectrum}

In a final set of test cases, the energy content associated with a number of random sea states is further observed. The two transfer functions under consideration are the theoretical force transfer function and a recent empirical calibration of the ICL wave basin. A uni-directional random sea state based upon a JONSWAP spectrum with $f_{p}=46 / 64 \mathrm{~Hz}, \gamma=2.3$ and $H_{s} \approx 0.02 \mathrm{~m}$ is generated with wave propagation directions of $\alpha=0^{\circ}, 20^{\circ}$ and $40^{\circ}$. In accordance with the discussion relating to the amplitude content (§3.1), the experimental results illustrated in Figure 15 (a)-(c) have been obtained by means of a reflection analysis on data recorded at eight spatial locations $(3.2 \mathrm{~m} \leq x \leq 4.6 \mathrm{~m}, y=0$ with $\Delta x=0.2 \mathrm{~m})$.

Observing Figure 15 in detail, one could easily conclude that both approaches give a good representation of the target spectrum. Indeed, the similarity between the results is (once again) perhaps the most important point, emphasising the success of the proposed theoretical force transfer function. Nevertheless, some interesting differences between the two transfer functions arise, not least because they are based on an entirely different approach. At first sight, the match between the experimental data and the target energy content seems superior when the generation is based on the theoretical force transfer function. However, this is to 
be expected since, following the reflection analysis, only the incident wave energy is considered. In contrast, an empirical calibration incorporates the beach reflections and seeks to achieve a desired energy content at a particular location in the wave basin.

Within any physical model study the test area of the wave basin will be subjected to both incident and reflected wave energy. As a result, it remains an open question as to which transfer function would model a desired sea state in a more realistic manner. Indeed the purpose of the present paper is not to recommend one particular approach, but rather to demonstrate that the proposed theoretical force transfer function can be used to provide an accurate representation of a desired sea state, without the need for extensive preliminary calibration. However, it must be borne in mind that beach reflections will inevitably be important for part of the desired frequency range. With an empirical calibration approach, it is possible to partly incorporate these reflections within the transfer function. However, if such an approach is adopted the wave energy will not necessarily be travelling in the desired directions. Difficulties of this type emphasise the need to reduce unwanted reflections to an absolute minimum.

\section{Conclusions}

A theoretical first-order force transfer function appropriate to the generation of multi-directional waves in wave basins has been derived. Based on this transfer function an extensive laboratory study has been undertaken. First, a number of uni-directional JONSWAP spectra propagating at different angles were generated. While the agreement with the target spectral content was found to be excellent for the longer wave components, some significant departures arose for the shorter wave components, particularly when propagating at large angles. This is shown to be caused (in large part) by the finite wavemaker segment width. In an attempt to overcome this apparent shortcoming, an ad-hoc procedure, incorporating the finite segment width, was introduced. Overall, the experimental data based on this ad-hoc approach gives consistently good agreement with the target spectral content.

In a second step, the generation of various focused wave events has shown that the correct application of the phase information led to the focusing of the individual wave components at the desired location in both space and time. This investigation includes both uni-directional and directionally spread focused wave groups. The data has been directly compared to a previously published empirical calibration; very good agreement between the two approaches and a linear prediction being demonstrated.

In a final step, the energy distribution associated with a random sea state has been further investigated. For this purpose, experimental data based upon the new theoretical force transfer function has been directly compared to data based upon a recent empirical calibration of the ICL wave basin. This latter comparison shows good agreement but also illustrates some interesting differences between the two approaches. In particular, the effect of beach reflections, which inevitably contaminate the testing area after a short period of time, has been noted, and differences between the two transfer functions have been explained.

\section{References}

Biésel, F. \& Suquet, F. (1954), 'Laboratory wave generating apparatus', St. Anthony Falls Hydraulic Laboratory, University of Minnesota, Minneapolis Project Report No. 39.

Boccotti, P. (1983), 'Some new results on statistical properties of wind waves', Applied Ocean Research 5, 134-140.

Chatry, G., Clement, A. H. \& Gouraud, T. (1998), Self-adaptive control of a piston wave absorber, in 'Proceedings of the Eighth International Offshore and Polar Engineering Conference', Vol. 1, pp. 127133.

Dalrymple, R. A. (1985), Physical Modelling in Coastal Engineering, Balkema.

Falnes, J. (2002), Ocean Waves and Oscillating Systems, Cambridge University Press.

Hald, T. \& Frigaard, P. (1997), Alternative method for active absorption in multi-directional waves, in 'Multi-directional Waves and their Interaction with Structures, 27th IAHR Congress', San Francisco, pp. 183-194. 
Hayden, D., Hoyt, J., Melendez, M., Moeller, H., Bargman, Y., Carpenter, S. \& Turner, S. (2010), Naval surface warfare center's wavemaker modernization program, in 'Proceedings of the 29th American Towing Tank Conference', Annapolis, MD, pp. 137-156.

Lin, C.-Y. \& Huang, C.-J. (2004), 'Decomposition of incident and reflected higher harmonic waves using four wave gauges', Coastal Engineering 51(5-6), 395 - 406.

Lindgren, G. (1970), 'Some properties of a normal process near a local maximum', The Annals of Mathematical Statistics 41, 1870-1883.

Mansard, E., Manoha, B. \& Funke, E. (1997), A survey of multi-directional wave facilities, in 'Multidirectional Waves and their Interaction with Structures, 27th IAHR Congress', San Francisco, pp. 195229.

Naito, S. \& Nakamura, S. (1985), Wave energy absorption in irregular waves by feedforward control system., in 'IUTAM Symposium Lisbon', Berlin, West Ger, pp. 269 - 280.

Masterton, S. \& Swan, C. (2008), 'On the accurate and efficient calibration of a 3d wave basin', Ocean Engineering 35(8-9), 763 - 773.

Naito, S. (2006), 'Wave generation and absorption in wave basins: Theory and applications', International Journal of Offshore and Polar Engineering 16(2), 81-89.

Newman, J. (2010), 'Analysis of wave generators and absorbers in basins', Applied Ocean Research 32, 71-82.

Rogers, D., Jason, I. \& Spinneken, J. (2010), A new look at wave synthesis using multiple wave fronts, in 'Proceedings of the 29th Americal Towing Tank Conference', Annapolis, MD, pp. 51-58.

Salter, S. (1982), Absorbing wave-makers and wide tanks, in 'Directional Wave Spectra Applications', pp. 185-200.

Sand, S. (1979), Three-dimensional deterministic structure of ocean waves, Series paper 24, Technical University of Denmark.

Sand, S. E. \& Mynett, A. E. (1987), Directional wave generation and analysis, in 'Wave Analysis and Generation in Laboratory Wave Basins, 22nd IAHR Congress', Lausanne, pp. 209-235.

Schäffer, H. A. (1998), Some design aspects of an absorbing 3D wavemaker, in 'Proceedings of the 26th International Conference on Coastal Engineering', Denmark, pp. 1082-1095.

Schäffer, H. A. \& Klopman, G. (1997), Review of multidirectional active wave absorption methods, in 'Multidirectional Waves and their Interaction with Structures, 27th IAHR Congress', San Francisco, pp. 159182.

Schäffer, H. A. \& Klopman, G. (2000), 'Review of multidirectional active wave absorption methods', Journal of Waterway, Port, Coastal and Ocean Engineering 126(2), 88 - 97.

Schäffer, H. A. \& Skourup, J. (1996), Active absorption of multidirectional waves, in 'Proceedings of the 25th International Conference on Coastal Engineering', USA, pp. 55-66.

Schäffer, H. A. \& Steenberg, C. (2003), 'Second-order wavemaker theory for multidirectional waves', Ocean Engineering 30(10), 1203 - 1231.

Spinneken, J. \& Swan, C. (2009a), 'Second-order wave maker theory using force-feedback control. Part I: A new theory for regular wave generation', Ocean Engineering 36(8), 539 - 548.

Spinneken, J. \& Swan, C. (2009b), 'Second-order wave maker theory using force-feedback control. Part II: An experimental verification of regular wave generation', Ocean Engineering 36(8), 549 - 555.

Spinneken, J. \& Swan, C. (2009c), Wave generation and absorption using force-controlled wave machines, in 'Proceedings of the 19th International Offshore and Polar Engineering Conference', Vol. 3, Osaka, Japan, pp. 397-404. 
Spinneken, J. \& Swan, C. (2011), 'Theoretical transfer function for force-controlled wave machines', International Journal of Offshore and Polar Engineering 21, 169 - 177.

Tromans, P., Anaturk, A. \& Hagemeijer, P. (1991), A new model for the kinematics of large ocean wavesapplication as a design wave, in 'Proceedings of the 1st International Offshore and Polar Engineering Conference', Vol. 3, Edinburgh, UK, pp. 64-71.

Ursell, F., Dean, R. \& Yu, Y. (1960), 'Forced small-amplitude water waves: a comparison of theory and experiment', Journal of Fluid Mechanics 7, 33-52. 


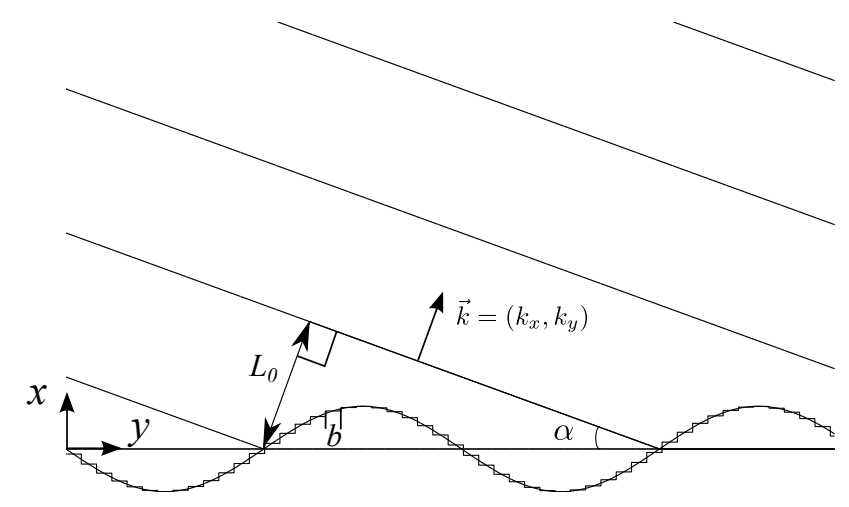

Figure 1: Layout of a 3D wave basin. Angled lines represent the crest contours of a progressive wave train propagating at an angle $\alpha$. Motion approximated by a piecewise constant function as adopted in a realistic segmented-type wavemaker.
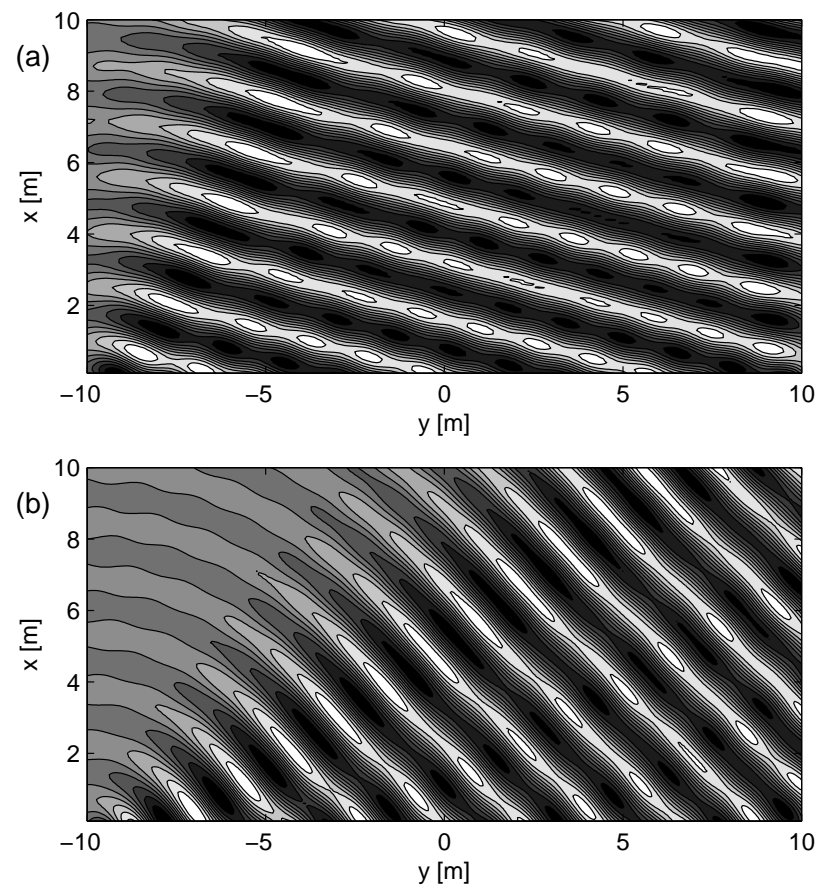

Figure 2: Normalised surface elevation contours for $h=1.5 \mathrm{~m}$ and $f=1.0 \mathrm{~Hz}$; (a) $\alpha=15^{\circ}$ and (b) $\alpha=45^{\circ}$; the wavemaker being located at $-10 \mathrm{~m} \leq y \leq 10 \mathrm{~m}$ 


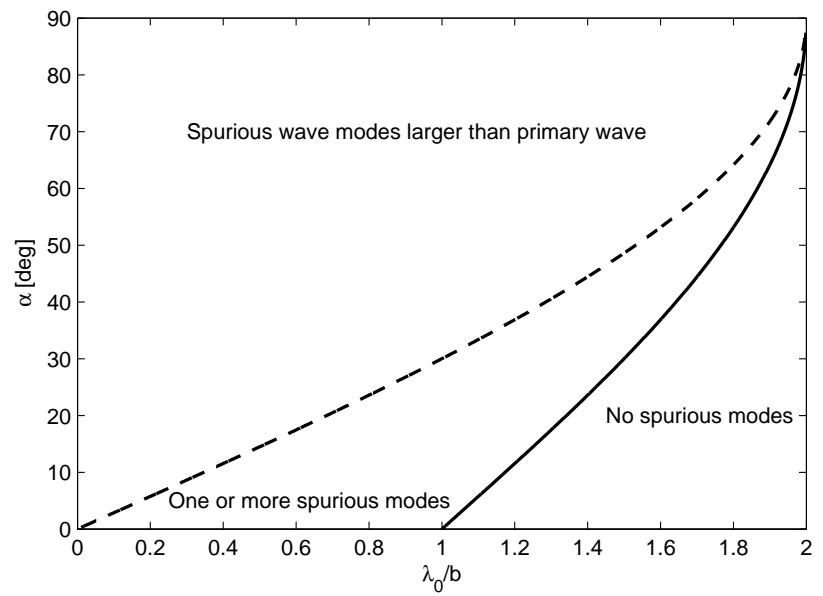

Figure 3: Spurious wave modes arising from the generation of oblique waves based on Sand and Mynett (1987); - Boundary between $p=0$ and $p \geq 1$, - Limiting case with $\varphi=\pi$. Note: This figure differs from that of Sand and Mynett (1987) in that they scaled their ordinate by $\sin \alpha$

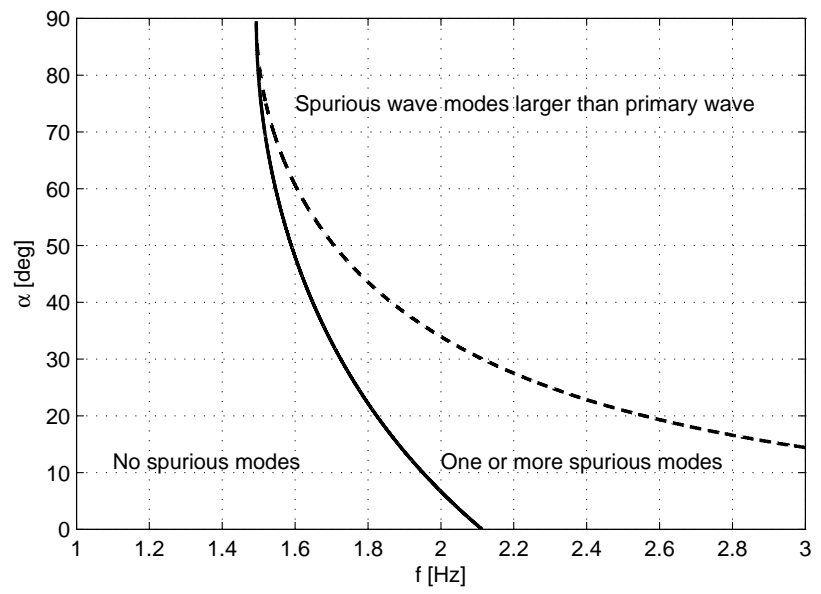

Figure 4: Spurious wave modes for the generation of oblique waves with $h=1.5 \mathrm{~m}$ and $b=0.36 \mathrm{~m}$; - Boundary between $p=0$ and $p \geq 1, \boldsymbol{-}$ Limiting case with $\varphi=\pi$ 


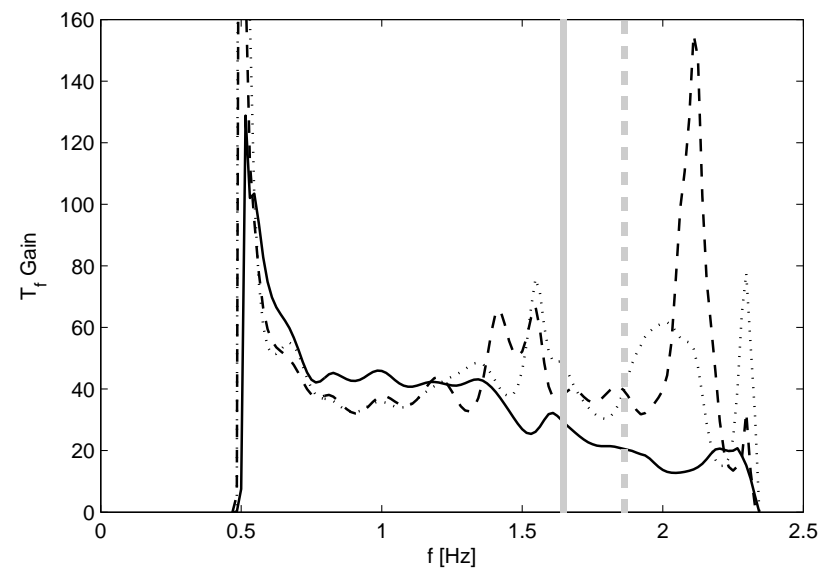

Figure 5: Empirical transfer function for a 3D wave basin, after Masterton \& Swan (2008); $\alpha=0^{\circ}$, - $\alpha=40^{\circ}, \cdots \alpha=-40^{\circ} ; \quad$ Limit for $p=0$ and $=-$ Limiting case with $\varphi=\pi$, both for $\alpha= \pm 40^{\circ}$
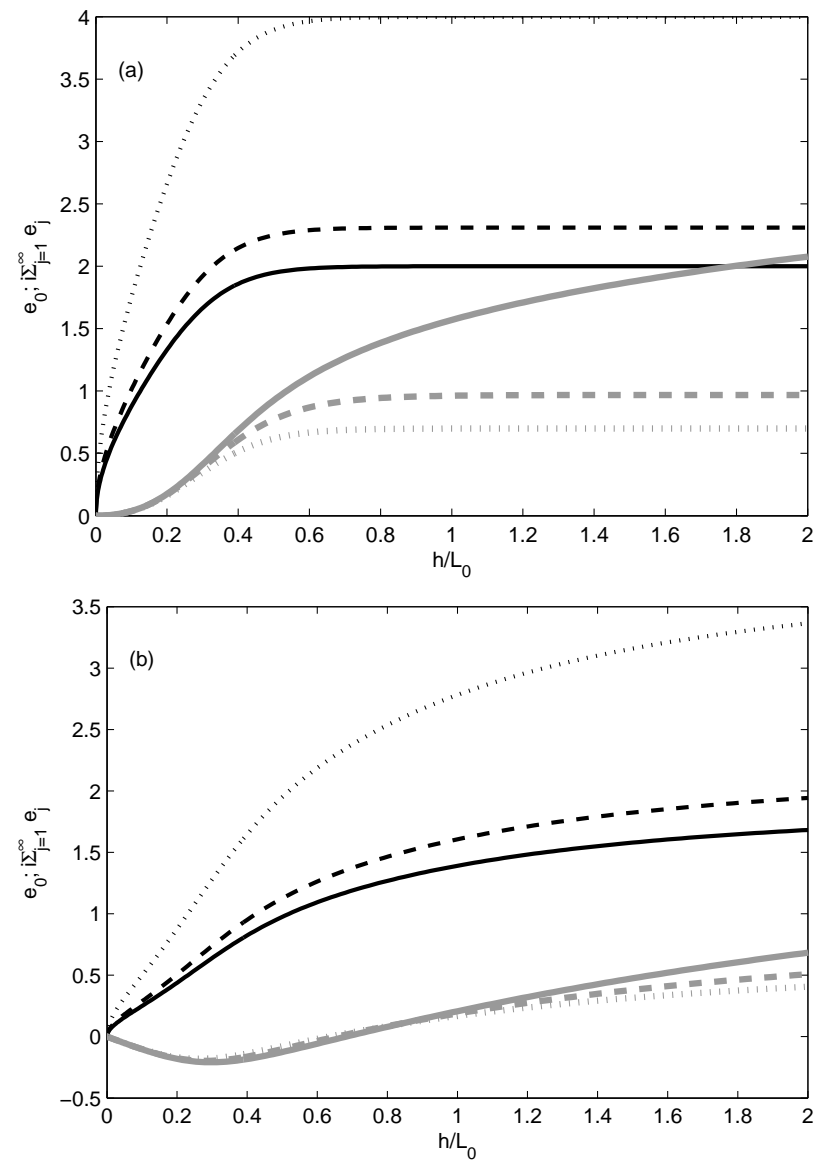

Figure 6: Dimensionless first-order coefficients. The black lines represent $e_{0}$ and the grey lines represent $i \Sigma_{j=1}^{\infty} e_{j} ; \longrightarrow \alpha=0^{\circ},-\boldsymbol{\alpha}=30^{\circ}, \cdots \alpha=60^{\circ}$. (a) Piston-type wavemaker and (b) flap-type wavemaker $(d / h=0.5)$. 

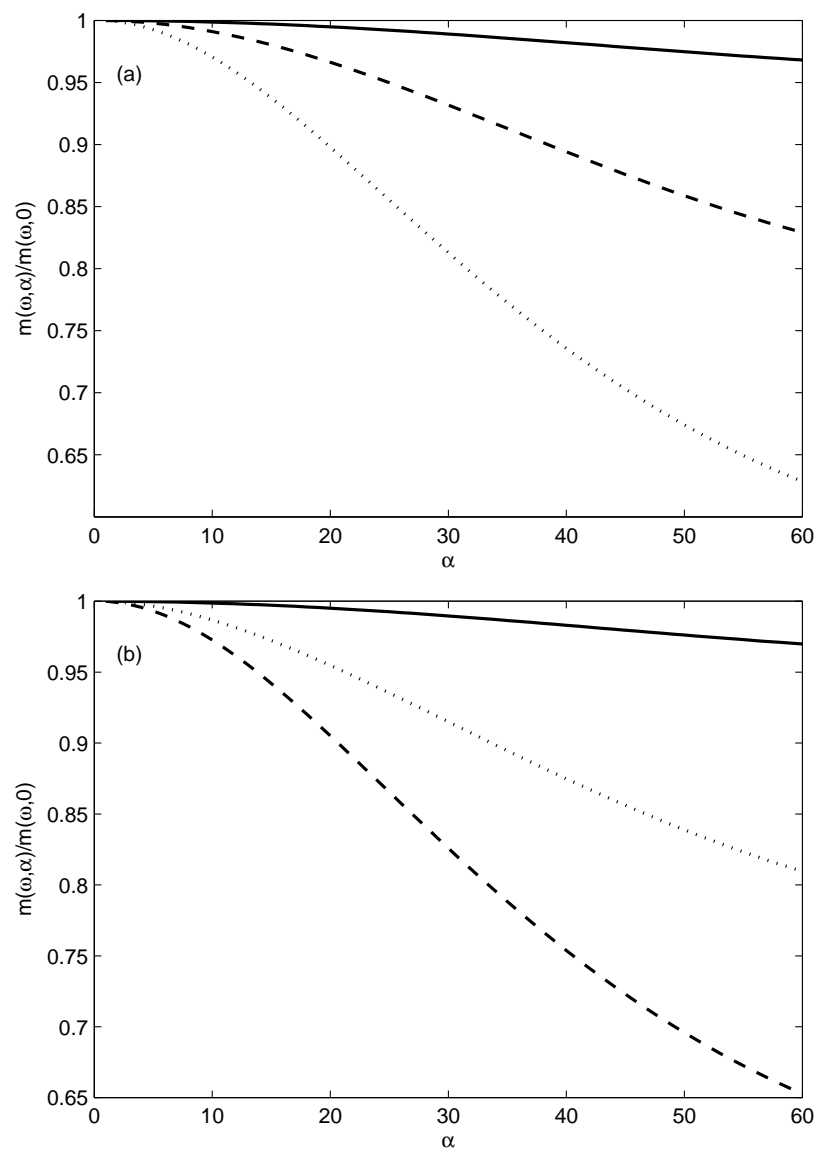

Figure 7: Added mass coefficient. $-h / L_{0}=0.1,-\boldsymbol{Z} h / L_{0}=0.3, \cdots h / L_{0}=0.5$. (a) Piston-type wavemaker and (b) flap-type wavemaker $(h / d=0.5)$

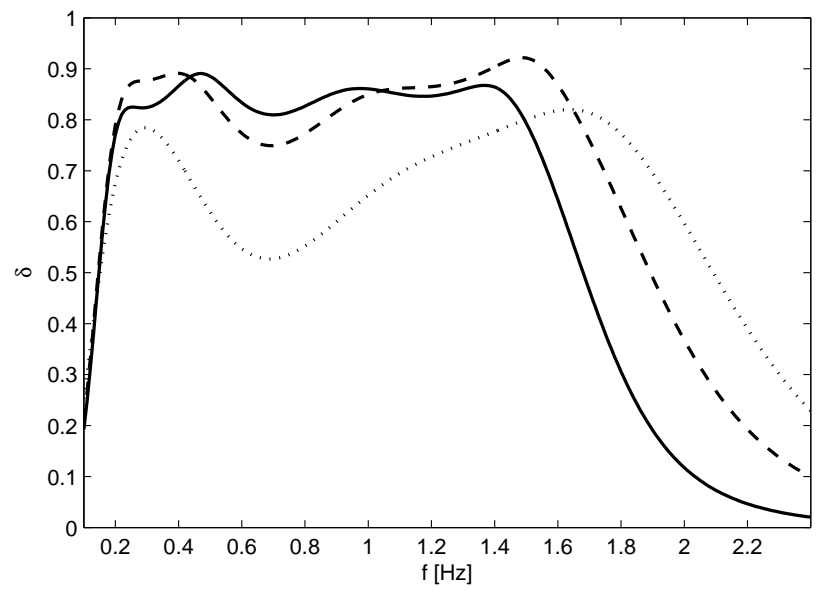

Figure 8: Amplitude absorption coefficient for a flap-type wavemaker $(d / h=0.533)$ in a wave basin with $h=1.5 \mathrm{~m}$; the controller being optimised for $\alpha=0^{\circ} ;-\alpha=0^{\circ},-\boldsymbol{-} \alpha=30^{\circ}, \cdots \quad \alpha=60^{\circ}$. Note: A coefficient of $\delta=1$ corresponds to perfect (100\%) wave absorption. 


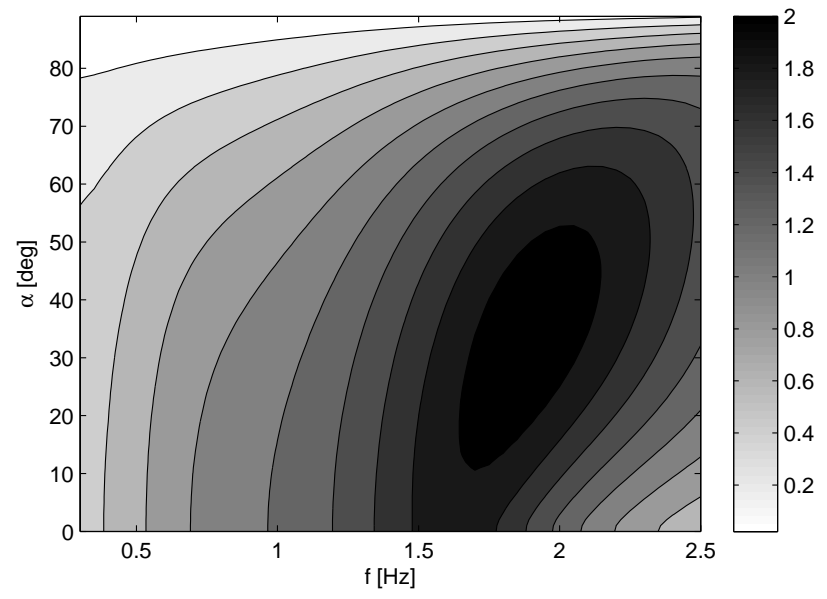

Figure 9: Contour plot representing the magnitude of the theoretical first-order force transfer function for the wave basin at ICL, $\left|c_{f}(\omega, \alpha)\right|$, in $[\mathrm{mm} / \mathrm{Nm}]$ 

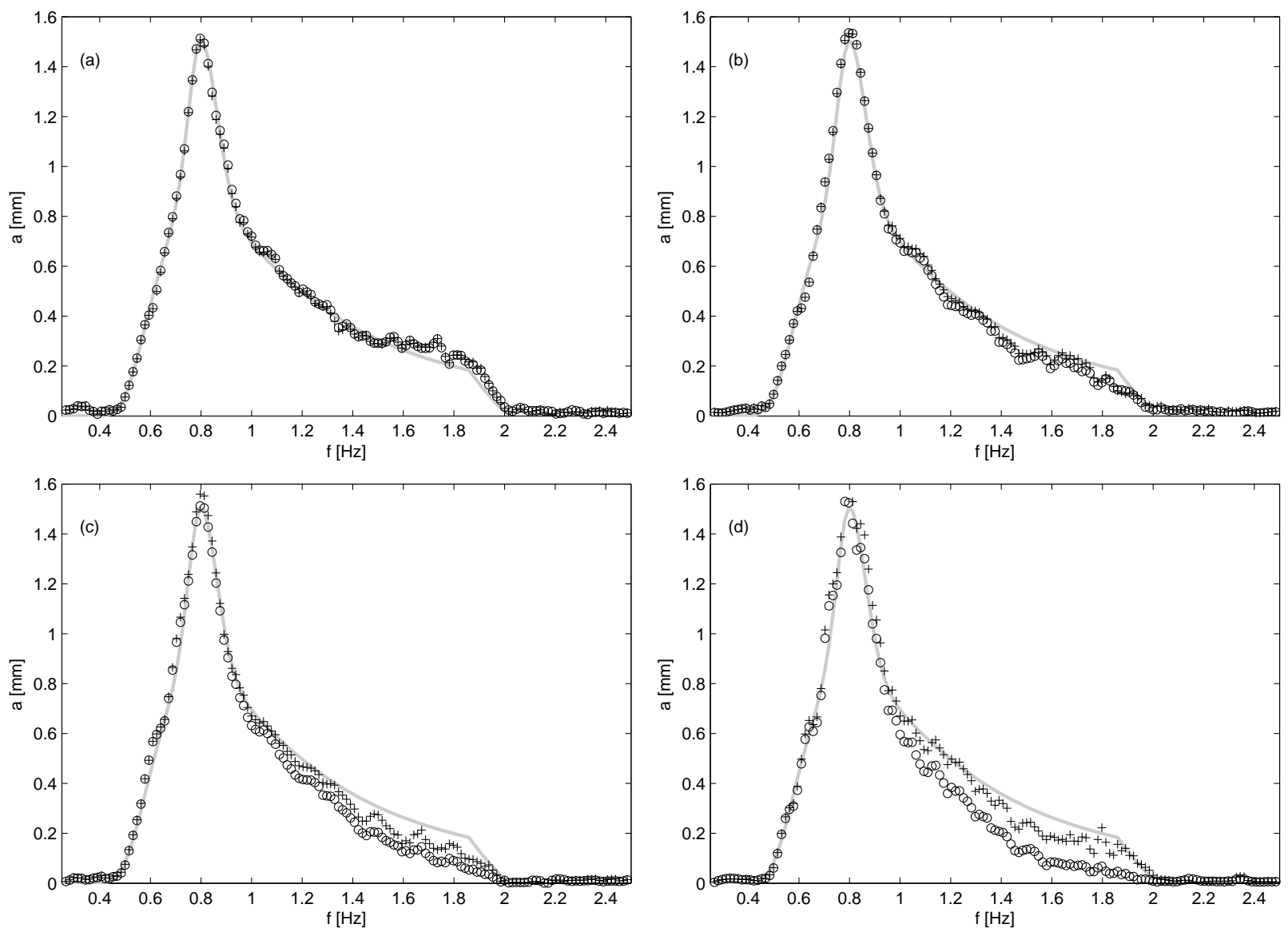

Figure 10: Amplitude content for a random sea state based upon a uni-directional JONSWAP spectrum with $f_{p}=51 / 64 \mathrm{~Hz}, \gamma=3.3$ and $H_{s} \approx 0.02 \mathrm{~m}:=\quad$ target amplitude spectrum, o theoretical force transfer function $c_{f}(\omega, \alpha),+a d$-hoc approach. Wave propagating at (a) $\alpha=0^{\circ}$, (b) $\alpha=15^{\circ}$, (c) $\alpha=30^{\circ}$ and (d) $\alpha=45^{\circ}$ 

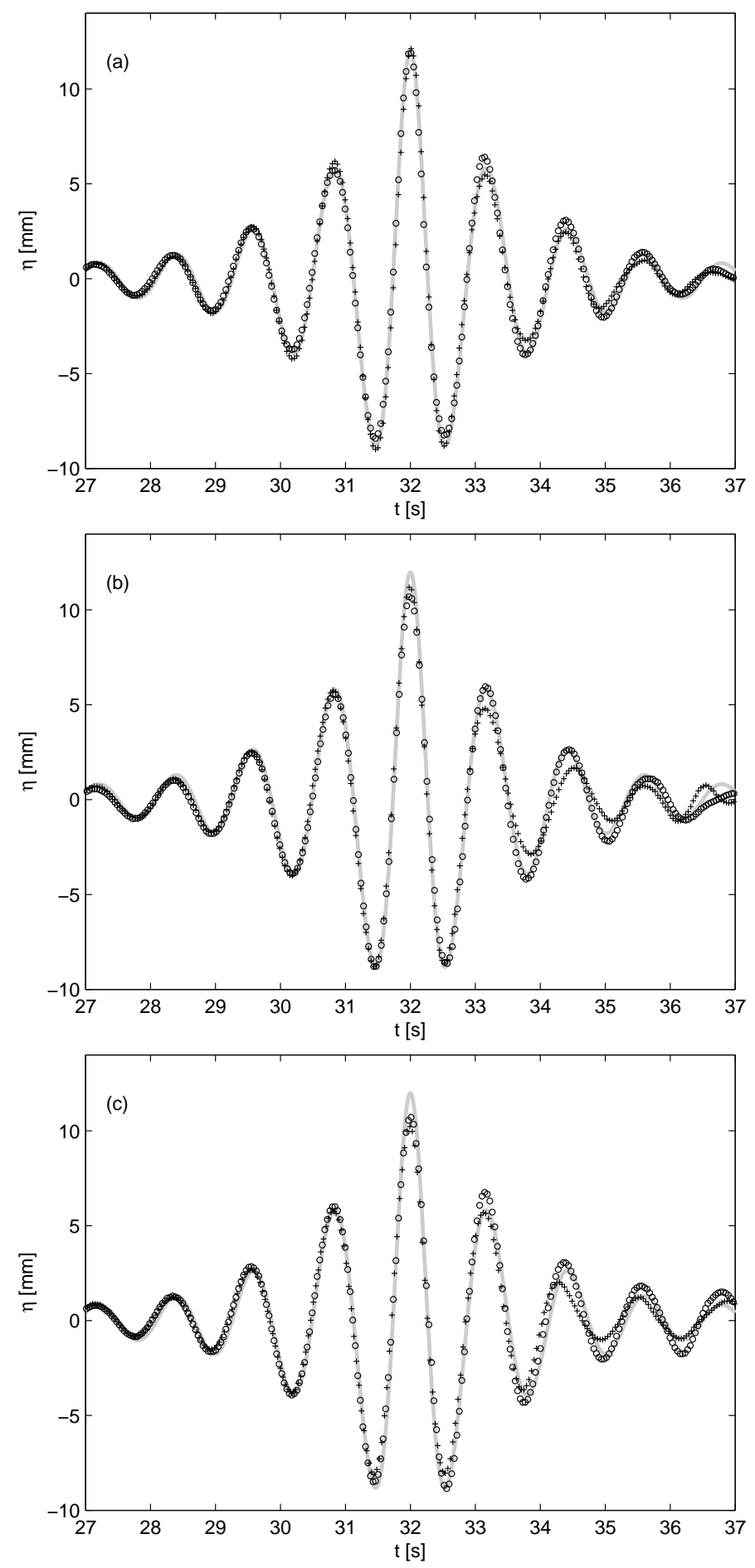

Figure 11: Surface profile for a uni-directional NewWave group based upon a JONSWAP spectrum with $f_{p}=53 / 64 \mathrm{~Hz}, \gamma=2.5, A=12 \mathrm{~mm}$; measured and focused at $x=4.0 \mathrm{~m}, y=0$. Theoretical (linear) solution; Experimental data based on o theoretical force transfer function, + empirical transfer function after MS. Wave propagating at (a) $\alpha=0^{\circ}$, (b) $\alpha=15^{\circ}$ and (c) $\alpha=30^{\circ}$. 

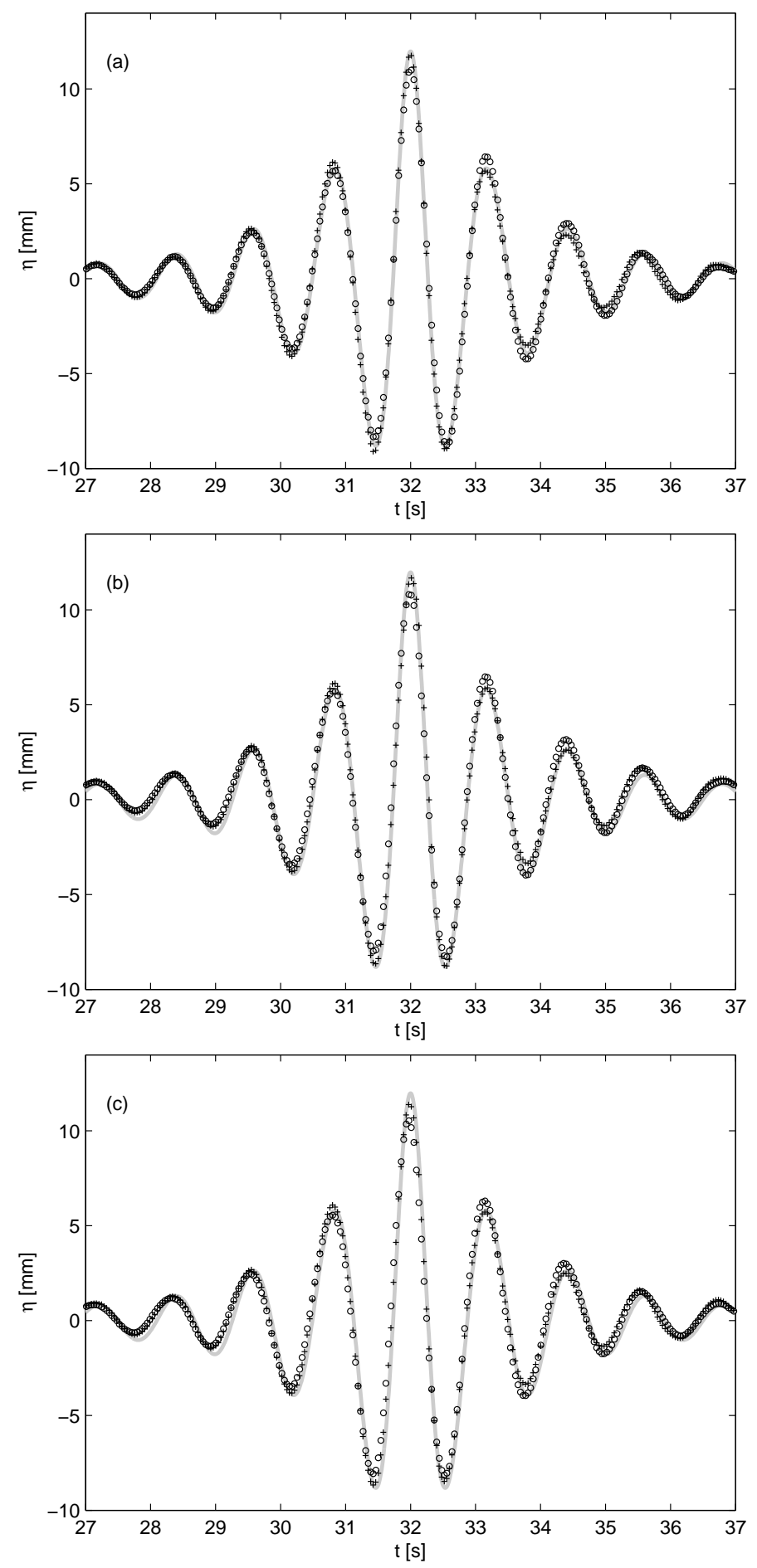

Figure 12: Surface profile for a multi-directional NewWave group based upon a JONSWAP spectrum with $f_{p}=53 / 64 \mathrm{~Hz}, \gamma=2.5, A=12 \mathrm{~mm}$ and $\sigma_{\alpha}=30^{\circ}$. Theoretical (linear) solution; Experimental data based on $\circ$ the theoretical force transfer function and + the empirical transfer function of MS. Measured and focused at $x=4.0 \mathrm{~m}$ and (a) $y=0$, (b) $y=-1.2 \mathrm{~m}$ and (c) $y=+1.2 \mathrm{~m}$. 


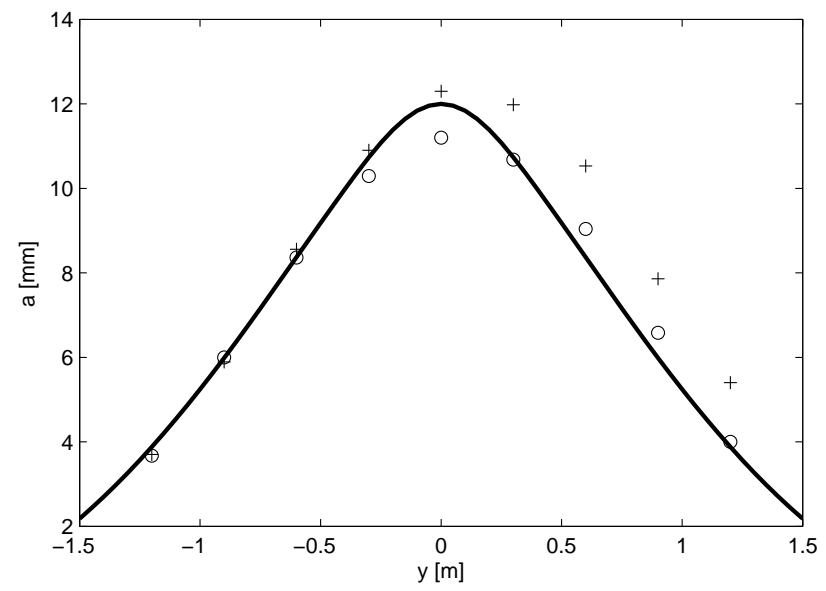

Figure 13: Transverse variation in the maximum crest elevation for a NewWave group based upon a JONSWAP spectrum with $f_{p}=53 / 64 \mathrm{~Hz}, \gamma=2.5, A=12 \mathrm{~mm}$ and $\sigma_{\alpha}=30^{\circ}$; focused at $x=4.0 \mathrm{~m}, y=0$ and $t=32 \mathrm{~s}$; Theoretical (linear) solution; Experimental data based on $\circ$ the theoretical force transfer function and + the empirical transfer function of MS

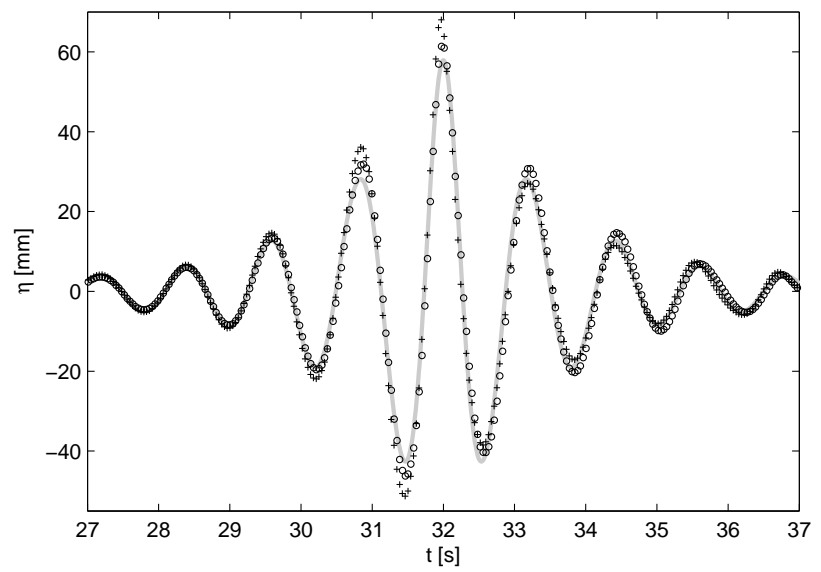

Figure 14: Surface profile for a multi-directional JONSWAP NewWave spectrum with $f_{p}=53 / 64 \mathrm{~Hz}, \gamma=2.5$, $A=58 \mathrm{~mm}$ and $\sigma_{\alpha}=30^{\circ}$; measured and focused at $x=4.0 \mathrm{~m}$ and $y=0$; Theoretical (linear) solution; Experimental data based on $\circ$ the theoretical force transfer function and + the empirical transfer function of MS 

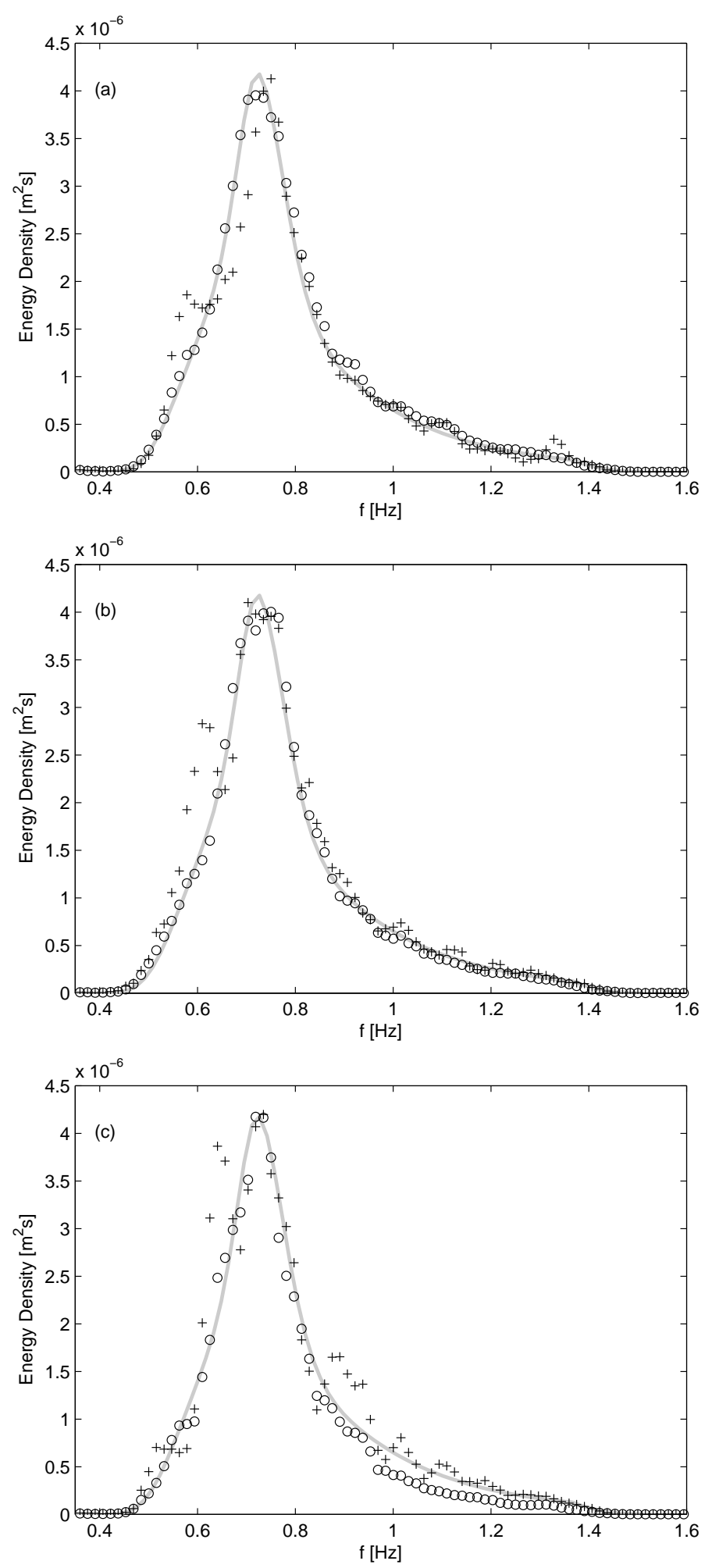

Figure 15: Energy content for a uni-directional JONSWAP spectrum with $f_{p}=46 / 64 \mathrm{~Hz}, \gamma=2.3$ and $H_{s} \approx 0.02 \mathrm{~m}$. Target (linear) spectrum; ○ data based upon the theoretical force transfer function $c_{f}(\omega, \alpha)$; and + data based upon a recent empirical transfer function for the ICL wave basin. Waves generated at (a) $\alpha=0^{\circ}$, (b) $\alpha=20^{\circ}$ and (c) $\alpha=40^{\circ}$. 